\title{
A Single-Component Photorheological Fluid with Light-Responsive Viscosity
}

A Single-Component

Photorheological Fluid with a

Light-Responsive Viscosity

Received 00th January 20xx

Accepted 00th January 20xx

DOI: $10.1039 / x 0 x \times 00000 x$

\author{
Elaine A. Kelly, ${ }^{a}$ Niamh Willis-Fox, ${ }^{b}$ Judith E. Houston, ${ }^{c}$ Camille Blayo, ${ }^{d}$ Giorgio Divitini, ${ }^{a}$ Nathan \\ Cowieson, ${ }^{e}$ Ronan Daly ${ }^{b}$ and Rachel C. Evans*a
}

\begin{abstract}
Viscoelastic fluids whose rheological properties are tunable with light have the potential to deliver significant impact in fields relying on a change in flow behavior, such as in-use tuning of combined efficient heat-transfer and drag-reduction agents, microfluidic flow and controlled encapsulation and release. However, simple, single-component systems must be developed to allow integration with these applications. Here, we report a single-component viscoelastic fluid, capable of a dramatic light-sensitive rheological response, from a neutral azobenzene photosurfactant, 4-hexyl-4'butyloxymonotetraethylene glycol $\left(\mathrm{C}_{6} \mathrm{AzoOC}_{4} \mathrm{E}_{4}\right)$ in water. From cryo-TEM, small-angle X-ray scattering and rheology measurements, we observe that the photosurfactant forms an entangled network of wormlike micelles in water, with a high viscosity (28 Pa.s) and viscoelastic behaviour. UV irradiation of the surfactant solution creates a less dense micellar network, with some vesicle formation. As a result, the solution viscosity is reduced by four orders of magnitude (to $1.2 \times 10^{-3} \mathrm{~Pa} \cdot \mathrm{s}$ ). This process is reversible and the high and low viscosity states can be cycled several times, through alternating UV and blue light irradiation.
\end{abstract}

\section{Introduction}

Smart viscoelastic fluids have the ability to change their rheological properties on-demand. ${ }^{1-8}$ These fluids have industrial applications in drag-reduction ${ }^{9-11}$ and efficient heattransfer ${ }^{12-14}$ and could deliver innovation in biomedicine (e.g. controlled encapsulation, ${ }^{15-18}$ drug delivery, ${ }^{19,20}$ tissue engineering ${ }^{21-23}$ ) microfluidic flow ${ }^{24}$ and materials templating. ${ }^{25-28}$ Viscoelastic fluids can be created using surfactants which self-assemble into an entangled micellar network above a critical concentration. As the self-assembly process is very sensitive, depending on the formulation, the micellar network and its related rheological properties can be disrupted by changes to the local environment, such as solution $\mathrm{pH}^{6}$ temperature,,$^{4,7}$ salinity, ${ }^{1,29,30}$ redox potential ${ }^{3}$ and electric ${ }^{31}$ or magnetic fields. ${ }^{32}$ However, these stimuli are often non-specific, can require invasive perturbation of the fluid, often alter the solution composition and may be slow to trigger a response. A more elegant way to perturb the micellar network is to use light. As a stimulus, light is a highly effective as it can be applied selectively, rapidly and remotely, and the wavelength, intensity and spatial localisation can be precisely controlled. The only requirement for use is that the viscoelastic fluid absorbs light.

\footnotetext{
a. Department of Materials Science and Metallurgy, University of Cambridge, 27 Charles Babbage Rd, Cambridge CB3 OFS, UK

${ }^{b}$ Institute for Manufacturing, Department of Engineering, University of Cambridge 17 Charles Babbage Rd, Cambridge CB3 OFS, UK

c European Spallation Source ERIC, Box 176, SE-221 00 Lund, Sweden

${ }^{d}$ School of Chemistry, Trinity College Dublin, College Green, Dublin 2, Ireland

e Diamond Light Source, Harwell Science and Innovation Campus, Didcot,

Oxfordshire, OX11 ODE, United Kingdom

*Corresponding author email: rce26@cam.ac.uk
}

Electronic Supplementary Information (ESI) available: synthesis and characterisation, photostationary state composition, additional rheology data, packing parameter calculations, structural parameters from model fitting to smallangle scattering data, additional cryo-TEM images. Supplementary videos available: flow behaviour before (Video S1) and after (Video S2) UV irradiation. See DOI: $10.1039 / \mathrm{x} 0 \times x \times 0000 \mathrm{x}$
There are two main approaches to creating photorheological fluids. First, and most commonly used, is the incorporation of a light-sensitive additive into a system already known to present viscoelastic behaviour.1,2,33-37 This method requires the creation of multicomponent formulations, generally a cationic surfactant, a salt, and light-responsive additive(s). It therefore overlooks the key benefit of light as a stimulus - its simplicity and preservation of a constant solution composition. The second, and less common approach is to create an entangled micellar network from a surfactant that is inherently light-responsive. Single-component systems are desirable as extra components may limit the compatibility of the photorheological fluid for a given application. For example, the need for a salt-based solution could lead to issues with salt deposition, surface fouling and solution salt depletion. ${ }^{38-40}$

Single-component photorheological fluids can be created by incorporating an azobenzene chromophore into a surfactant structure. Azobenzene photosurfactants (AzoPS) retain the intrinsic photoisomerisation response of the azobenzene unit, switching from the linear, hydrophobic trans-isomer to the bent, more hydrophilic cis-isomer upon application of light of the correct frequency. ${ }^{41-44}$ The trans-isomer can be recovered upon irradiation at longer wavelengths or gentle heating. This leads to stark differences in the self-assembly behaviour for each isomer, which can propagate to changes in bulk solution properties, including viscoelasticity and viscosity. There have been limited examples of photorheological fluids based on AzoPS to date. Peng et al. reported a polyethylene glycol-based AzoPS that could reversibly reduce its viscosity from either 2000 to $10 \mathrm{~Pa} \cdot \mathrm{s}$ ( $50 \mathrm{wt} \%$ in water) or 45 to $4 \mathrm{~Pa} \cdot \mathrm{s}$ ( $30 \mathrm{wt} \%$ in water) upon UV irradiation. ${ }^{45}$ This behaviour was attributed to the formation of lyotropic liquid crystal (LLC) phases (hexagonal or lamellar, respectively), which were disrupted to an isotropic phase upon photoisomerisation. Using lower surfactant concentrations, Song et al. reported a single-component photorheological fluid based on an ionic, gemini azobenzene surfactant $\left(\mathrm{C}_{12}-\mathrm{Azo}-\mathrm{C}_{12}\right) .{ }^{46}$ Reversible switching between low ( 0.01 Pa.s) and high (733.8 Pa.s) viscosity states was observed 
for a $60 \mathrm{mM}$ (3.9 wt\%) aqueous AzoPS solution, using UV and blue light, respectively. Here, we report a neutral AzoPS, $\mathrm{C}_{6} \mathrm{AzoOC}_{4} \mathrm{E}_{4}$, (4-hexyl-4'butyloxymonotetraethylene glycol azobenzene, Figure 1a) which, in aqueous solution at low concentration (48 mM, $2.5 \mathrm{wt} \%)$, can reversibly switch its viscosity four orders of magnitude, between a non-viscous $\left(1.2 \times 10^{-3} \mathrm{~Pa} \cdot \mathrm{s}\right)$ solution and a viscous (28 Pa.s) viscoelastic state, upon UV and blue light irradiation, respectively. To the best of our knowledge, this is the first report of a singlecomponent photorheological fluid created using a neutral AzoPS at low concentrations. Neutral surfactants offer the benefit of lower toxicities and a lower critical micelle concentration compared to their charged counterparts, meaning that less material is required for a given use.

\section{Experimental}

\section{Synthesis}

The synthesis of the $\mathrm{C}_{6} \mathrm{AzoOC}_{4} \mathrm{E}_{4}$ was achieved through modifications of previous literature procedures and has been reported elsewhere. ${ }^{44}$ Full experimental details are available in the Electronic Supplementary Information (ESI).

\section{Rheology}

Rheology measurements were performed on an Anton Paar MCR 302 rheometer. Samples were measured at $25^{\circ} \mathrm{C}$ using a cone-plate geometry $\left(50 \mathrm{~mm}\right.$ diameter, $1^{\circ}$ cone angle, 0.101 $\mathrm{mm}$ gap). An amplitude sweep was performed to ensure that the samples were in the linear response regime. Frequency sweeps to determine the storage $\left(G^{\prime}\right)$ and loss moduli $\left(G^{\prime \prime}\right)$ were measured at a $1 \%$ strain rate. Fits to a Maxwell Model (see Eq. S2 and S3, ESI) were performed using the software package RepTate. Rheology measurements were performed before and after 2.5 hours of UV irradiation and after 2 hours of blue light irradiation. Irradiation was performed using either UV (365 nm, $3.7 \mathrm{~mW} \mathrm{~cm}^{-2}$ at $4 \mathrm{~cm}$ from source) or blue light (465 nm, $1.3 \mathrm{~mW} \mathrm{~cm}^{-2}$ at $4 \mathrm{~cm}$ from source) LEDs in a custom built lightbox. Conversion to the cis- or trans-PSS was confirmed using UV-Vis absorption spectroscopy (Figure S1).

\section{Birefringence}

Photographs were taken of $\mathrm{C}_{6} \mathrm{AzoOC}_{4} \mathrm{E}_{4}$ (2.5 wt\% in water) between crossed polarisers, before and after 2.5 hours UV irradiation.

\section{Absorption Spectroscopy}

UV-Vis absorption spectra were recorded on either a Perkin Elmer Lambda 35 spectrometer with a slit width of $0.5 \mathrm{~nm}$ or a Perkin Elmer Lambda 750 spectrophotometer using wavelength scan with a resolution of $1 \mathrm{~nm}$ at a scan speed of $1 \mathrm{~nm} / \mathrm{s}$ and a slit width of $2 \mathrm{~nm}$. Measurements were carried out in quartz cells with a $1 \mathrm{~cm}$ path length. All samples were prepared in deionised water.

\section{Chemical Structure Modelling Engine}

Three-dimensional (3D) models of chemical structures were created using the PerkinElmer Informatics software Chem3D. The algorithm is based on modified molecular mechanics, which consider bond stretching, angle bending, internal rotation and van der Waals interactions. While this is not a full-scale molecular mechanics engine, it produces a reasonable estimation of the molecular conformation

\section{Cryo-TEM}

Samples were vitrified using a Thermofisher Vitrobot in a controlled environment $\left(4^{\circ} \mathrm{C}, 100 \%\right.$ relative humidity). Transdominant samples $(0.5 \mathrm{wt} \%$ ) were prepared under ambient light while cis-dominant samples (0.5 wt\%) were irradiated with UV light ( $365 \mathrm{~nm}, 3.7 \mathrm{~mW} \mathrm{~cm}^{-2}$ at $4 \mathrm{~cm}$ from source) for 90 min prior to vitrification. This lower concentration required a shorter UV irradiation time for conversion to the cis-PSS compared to the highly absorbing 2.5 wt\% sample used for rheology measurements. A small volume $(2.5 \mu \mathrm{L})$ of AzoPS solution (0.5 wt\% in water) was deposited on a Quantifoil 1.2/1.3 holey carbon film. The sample was blotted for $2.5 \mathrm{~s}$ and plunged into liquid ethane to form the vitrified specimens. Specimens were stored in liquid nitrogen $\left(-196^{\circ} \mathrm{C}\right)$ until imaging. The vitrified specimens were imaged with a ThermoFisher Krios G3i at 300kV acceleration voltage, using a Falcon 3 camera.

\section{Small-angle X-ray Scattering}

Synchrotron SAXS measurements were performed on the BioSAXS beamline B21, Diamond Light Source, Harwell, United Kingdom. B21 operates in a fixed sample to detector distance $(4.014 \mathrm{~m})$ with $\mathrm{X}$-ray beam energy $E=12.4 \mathrm{keV}$ giving a $q$-range of 0.031-3.8 $\mathrm{nm}^{-1}$. Samples were loaded into PCR well plates in an automated sample changer. Samples $(30 \mu \mathrm{L})$ were then delivered into a temperature-controlled quartz capillary and exposed for $3 \mathrm{~s}$, collecting 60 frames at $20^{\circ} \mathrm{C}$. Data were collected using a Pilatus Dectris $2 \mathrm{M}$ detector. The background was manually subtracted using ScÅtter. All model fitting was performed using SASview (4.2.2). ${ }^{47}$

\section{Results and Discussion}

We first probed the photoisomerisation of $\mathrm{C}_{6} \mathrm{AzoOC}_{4} \mathrm{E}_{4}$ in water using UV-Vis absorption spectroscopy and found that it reversibly isomerises between $84 \%$ cis and $95 \%$ trans photostationary states (PSS) upon illumination with UV (365 $\mathrm{nm}$ ) and blue (465 $\mathrm{nm}$ ) light, respectively (Figure 1b). Samples were irradiated until no further changes were observed in the absorption spectra. It should be noted that it is not possible to obtain $100 \%$ conversion from the trans- to the cis- isomer due to the overlap of their respective absorption spectra, with cis and trans-dominant PSS formed instead (see ESI for PSS calculations). A $100 \%$ trans-isomer solution is recovered upon gentle heating at $60^{\circ} \mathrm{C}$. It was observed by eye that $2.5 \mathrm{wt} \%$ aqueous solutions of $\mathrm{C}_{6} \mathrm{AzoOC}_{4} \mathrm{E}_{4}$ in the native trans-state result in a gooey solution (ESI, Video S1) which changes to a watery fluid after UV illumination (ESI, Video S2). Steady-state shear and oscillatory rheology measurements were performed to quantify these observations. Before UV illumination, in the native trans-state, $\mathrm{C}_{6} \mathrm{AzoOC}_{4} \mathrm{E}_{4}$ is a non-Newtonian fluid with a high viscosity (>10 Pa.s) which demonstrates shear-thinning behaviour (Figure 2a). After UV irradiation (2.5 hours), the 
(a)
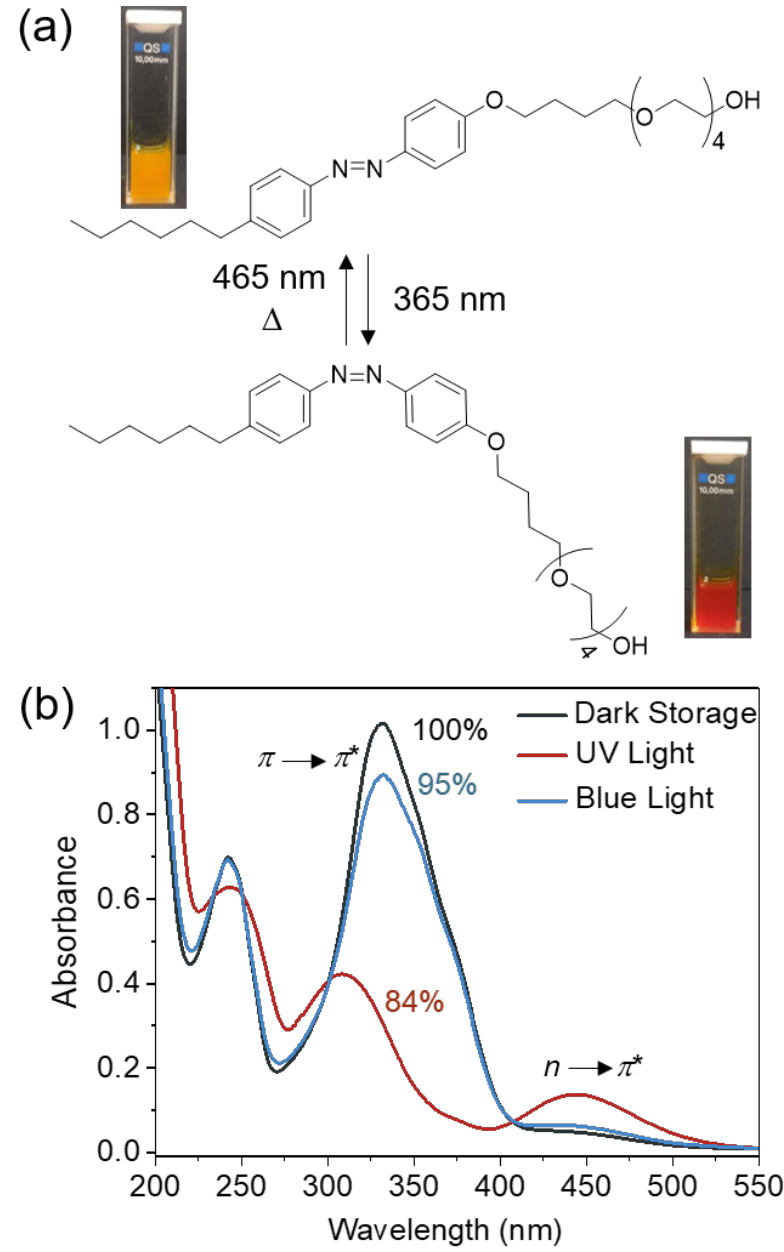

Figure 1. (a) Molecular structure of $\mathrm{C}_{6} \mathrm{AzoOC}_{4} \mathrm{E}_{4}$ in the trans and cis isomeric forms and photographs of $\mathrm{C}_{6} \mathrm{AzoOC}_{4} \mathrm{E}_{4}(2.5 \mathrm{wt} \%, 48 \mathrm{mM}$ in water) before and after UV irradiation. (b) UV-Vis absorption spectra of $\mathrm{C}_{6} \mathrm{AzoOC}_{4} \mathrm{E}_{4}(0.065 \mathrm{mM})$ in the native trans-state (black), after UV irradiation (30 sec) and formation of the cis-PSS (red) and after blue light irradiation ( $3 \mathrm{~min}$ ) and formation of the trans-PSS (blue).

$\mathrm{C}_{6} \mathrm{AzoOC}_{4} \mathrm{E}_{4}$ solution behaved as a low viscosity (0.0012 Pa.s), Newtonian fluid, with a difference in zero-shear viscosity of 4 orders of magnitude compared to the native-trans solution. UVVis absorption spectra recorded after rheology measurements confirmed that the sample remained in the cis-PSS over the course of the experiments (ESI, Figure S1). The non-Newtonian shear-thinning behaviour observed before UV illumination is characteristic of entangled chains disentangling and aligning themselves with the applied shear.7,48,49 There is no suggestion of entanglement present for the cis-PSS solution due to the low viscosity and lack of shear-dependent behaviour. When viewed under crossed-polarisers, birefringence can be observed for the native trans-state solution (Figure $2 \mathrm{~b}$ ). This strongly indicates the presence of wormlike micelles (WLM), the alignment of which under shear results in flow birefringence.8,50-53 After UV irradiation, no flow birefringence was observed, implying a change in the micellar structure and disappearance of WLM (Figure 2b).
The dynamic behaviour of $\mathrm{C}_{6} \mathrm{AzOOC}_{4} \mathrm{E}_{4}$ before UV illumination (native trans-state) was probed using oscillatory shear measurements. Figure $2 \mathrm{c}$ shows viscoelastic behaviour, signified by a crossover from between the storage $\left(G^{\prime}\right)$ and loss $\left(G^{\prime \prime}\right)$ moduli (from $G^{\prime}<G^{\prime \prime}$ to $G^{\prime}>G^{\prime \prime}$ ) as a function of angular frequency $(\omega)$, and the fits to a Maxwell model (see ESI Section 5, Eq. S3-S5 for details). A Cole-Cole plot further confirms this viscoelastic, Maxwellian behaviour. Deviation from the model at high frequencies is common and is attributed to fast relaxation processes such as Rouse or 'breathing' modes. ${ }^{48,54-56}$ The crossover frequency $\left(\omega_{c}\right)$ is inversely related to the relaxation time $\left(\tau_{\mathrm{R}}\right)$ of the solution, with a value of $28 \mathrm{~s}$ obtained here. ${ }^{56}$ For a Maxwell fluid, the zero-shear viscosity, $\eta_{0}$, is given by $\eta_{0}=G_{0} \tau_{R},{ }^{56}$ which results in a value of $28 \mathrm{~Pa} \cdot \mathrm{s}$ in this case. This is four orders of magnitude higher than the solution viscosity after UV irradiation $\left(1.2 \times 10^{-3} \mathrm{~Pa} \cdot \mathrm{s}\right)$. For comparison, the viscosity of water at $25{ }^{\circ} \mathrm{C}$ is $8.9 \times 10^{-4} \mathrm{~Pa}$.s. After UV illumination of $\mathrm{C}_{6} \mathrm{AzOOC}_{4} \mathrm{E}_{4}$ and formation of the low viscosity, non-Newtonian fluid, the possibility of recovering the initial high viscosity, viscoelastic behaviour was investigated. The cis$\mathrm{PSS}_{6} \mathrm{AzoOC}_{4} \mathrm{E}_{4}$ solution was irradiated with blue light ( 2 hours) to obtain the trans-PSS. Repeat steady-state and oscillatory shear measurements demonstrate that the initial viscosity and viscoelastic behaviour is fully recovered with blue light. (Figure 2a, ESI, Figure S2). Moreover, it was possible to reversibly cycle between low and high viscosity states by alternating UV and blue-light illumination (Figure 2d). for 2.5 and 2 hours, respectively.

The self-assembled structures formed by trans- and cis$\mathrm{C}_{6} \mathrm{AzoOC}_{4} \mathrm{E}_{4}$ were investigated using cryo-transmission electron microscopy (cryo-TEM) and small-angle X-ray scattering (SAXS). Figures $3 \mathrm{a}$ and $3 \mathrm{~b}$ show representative cryo-TEM images of $\mathrm{C}_{6} \mathrm{AzoOC}_{4} \mathrm{E}_{4}\left(0.5 \mathrm{wt} \%\right.$ in $\mathrm{H}_{2} \mathrm{O}$ ) before and after 90 minutes of UV irradiation. Further cryo-TEM images are available in the ESI (Figure S4). Figure $3 a$ shows the formation of a densely entangled network of elongated wormlike micelles for trans$\mathrm{C}_{6} \mathrm{AzoOC}_{4} \mathrm{E}_{4}$, in line with what would be expected based on the observed high viscosity, shear-thinning behaviour, flow birefringence and viscoelasticity.7,48,49,51 WLMs are cylindrical micelles which grow uniaxially along their length with increased surfactant concentration. The shape of the micelles formed by surfactants can be predicted using the packing parameter, $P=$ $V / a_{0} l$, where $V$ is the volume of the hydrophobic surfactant tail, $a_{0}$ is the equilibrium area of the surfactant head group and $l$ is the length of the hydrophobic surfactant tail. ${ }^{57}$ In this case, cylindrical structures are predicted for trans $-\mathrm{C}_{6} \mathrm{AzoOC}_{4} \mathrm{E}_{4}$, consistent with WLMs $(P=0.4,1 / 3<P<1 / 2$ is indicative of cylinder formation, ESI, Table S2). ${ }^{57,58}$ From the cryo-TEM images, the diameter of the WLMs is estimated to be $47.1 \pm$ $7.2 \AA$, roughly twice the value of the hydrophobic tail length of $\mathrm{C}_{6} \mathrm{AzoOC}_{4} \mathrm{E}_{4}(24.9 \AA$, ESI, Table S2). This indicates that the crosssectional radius of the cylindrical micelles is one surfactant monomer. From the cryo-TEM images, the length of the WLM is estimated to be on the order of $\mathrm{nm}-\mu \mathrm{m}$. 
(a)

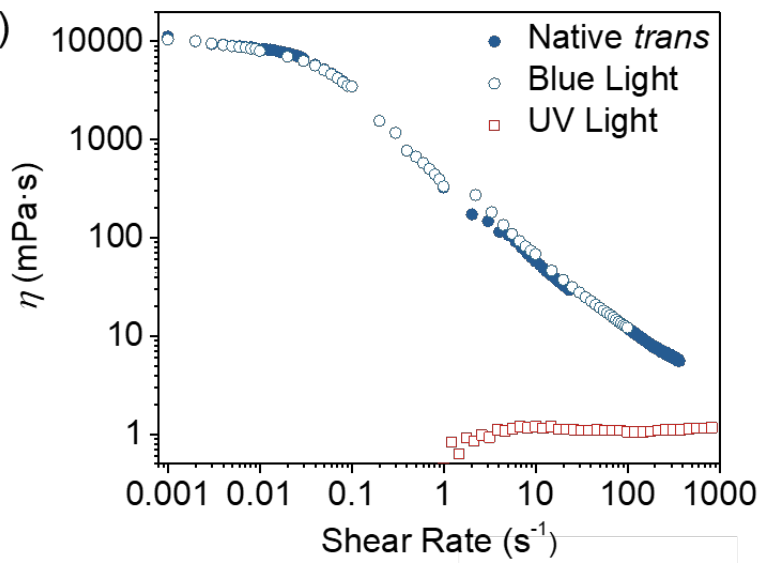

(c)

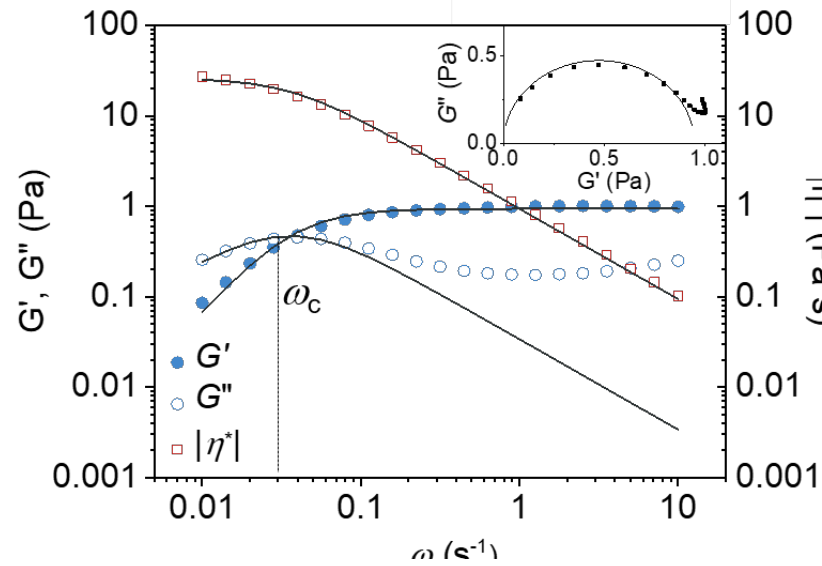

(b)
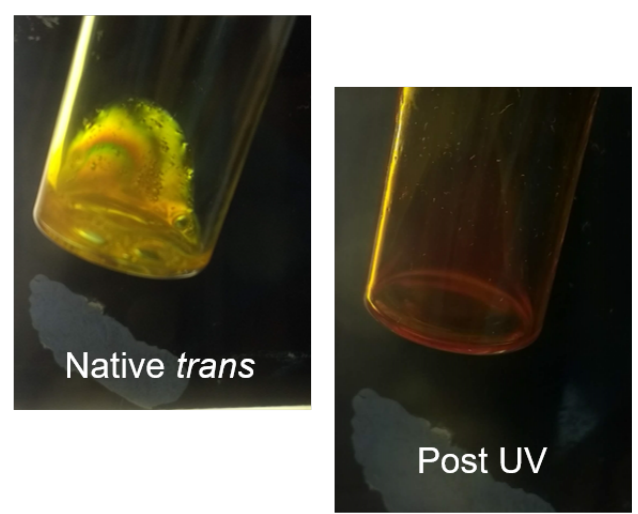

(d)

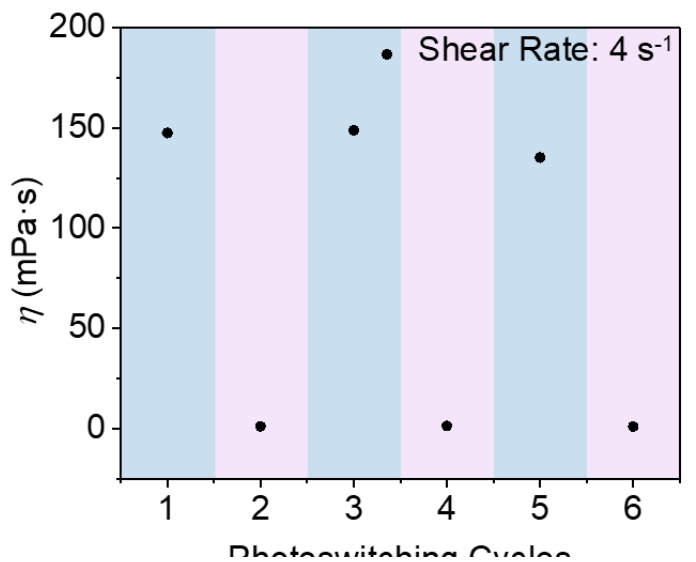

Figure 2. Photorheological characteristics of $\mathrm{C}_{6} \mathrm{AzoOC}_{4} \mathrm{E}_{4}$ (2.5 wt\% in water). (a) Variation of viscosity ( $\eta$ ) with increasing shear rate in the native state (navy filled circles), after UV irradiation (red open squares) and after blue-light irradiation (blue open circles). Photographs show a tilted petri-dish containing $\mathrm{C}_{6} \mathrm{AzoO}_{2} \mathrm{C}_{4} \mathrm{E}_{4}$ before (left) and after (right) UV irradiation. (b) Photographs of the flow birefringence of $\mathrm{C}_{6} \mathrm{AzOOC}_{4} \mathrm{E}_{4}$ in the native state (top) and the lack of birefringence after $2.5 \mathrm{~h} U \mathrm{~V}$ irradiation (bottom). (c) Variation of storage $\left(G^{\prime}\right.$, blue filled circles) and loss ( $G^{\prime \prime}$, blue open circles) moduli and complex viscosity ( $\eta^{*} \mid$, open squares) with angular frequency. Black lines are fits to a Maxwell model. Inset: A Cole-Cole plot traces a semi-circle with a radius equal to the crossover frequency $\left(\omega_{\mathrm{c}}\right)(\mathrm{d})$ Reversible cycling of the solution viscosity with alternating blue light and UV illumination, indicated by the blue and pink sectors, respectively

The SAXS profiles of native-state $\mathrm{C}_{6} \mathrm{AzoOC}_{4} \mathrm{E}_{4}(0.5 \mathrm{wt} \%, 2.5 \mathrm{wt} \%)$ decay as $q^{-1}$ in the low $q$ region, which indicates that cylindrical scattering bodies are present across this concentration range (Figure 3c). $48,59,60$ Figure $3 \mathrm{~b}$ shows cryo-TEM images of $\mathrm{C}_{6} \mathrm{AzoOC}_{4} \mathrm{E}_{4}(0.5 \mathrm{wt} \%)$ after 90 minutes irradiation with UV light. A visibly different network with a significantly lower density and fewer entanglements than that of the trans-isomer solution can be observed, with looping, branching and vesicle formation present. The packing parameter for cis- $\mathrm{C}_{6} \mathrm{AzoOC}_{4} \mathrm{E}_{4}$ is calculated to be 0.46 (ESI, Table S2), approaching the limit of cylinder formation and onset of vesicle formation $(1 / 2>P>1)$. The individual threads here have a diameter of $43.2 \pm 9 \AA$.

In general, for small-angle scattering the position of the first scattering minimum is correlated to the radius of the scattering bodies in solution. ${ }^{61-67}$ Here, the first minima shift to higher $q$ after UV irradiation (from 0.078 to $0.1 \AA^{-1}$ ) indicating that cylinders with a smaller radius are formed. There is not a significant difference in the shape of the scattering profiles before and after UV illumination, suggesting that on the length scales probed by SAXS $(2-200 \mathrm{~nm})$ the shape of the self- assembled structures is similar, with all decaying as $\mathrm{q}^{-1}$, indicative of cylindrical scattering bodies. (Figure 3c). All scattering profiles could be fitted using cylindrical models. Trans $-\mathrm{C}_{6} \mathrm{AzoOC}_{4} \mathrm{E}_{4}$ was best described using a cylinder model (dotted line, Figure $3 c$, radius: $46.9 \AA$, polydispersity: 0.27 ) at 0.5 wt\% and a flexible cylinder model (solid line, Figure $3 c$, radius: $58.9 \AA$, polydispersity: 0.27 ; Kuhn length: $618.2 \AA$, polydispersity: 0.15 ) at $2.5 \mathrm{wt} \%$. This change from cylinder to flexible cylinder model is consistent with the presence of wormlike micelles, which grow uniaxially along their length with increased concentration. After UV irradiation, at $0.5 \mathrm{wt} \%$ cis$\mathrm{C}_{6} \mathrm{AzoOC}_{4} \mathrm{E}_{4}$ could be described using a flexible cylinder model (solid line, Figure $3 c$, radius: $42.1 \AA$, polydispersity: 0.28 ; Kuhn length: $100.8 \AA)$.The existence of a photostationary state $(84 \%$ cis, $16 \%$ trans) after UV irradiation suggests a mixture of selfassembled structures is formed, which will distort the resulting scattering profile e.g. the presence of WLM and vesicles in the cryo-TEM after UV irradiation. At $2.5 \mathrm{wt} \%$, cis- $\mathrm{C}_{6} \mathrm{AzoOC}_{4} \mathrm{E}_{4}$ was described best by a flexible cylinder model, with a smaller radius than the trans-form (solid line, Figure $3 c$, 

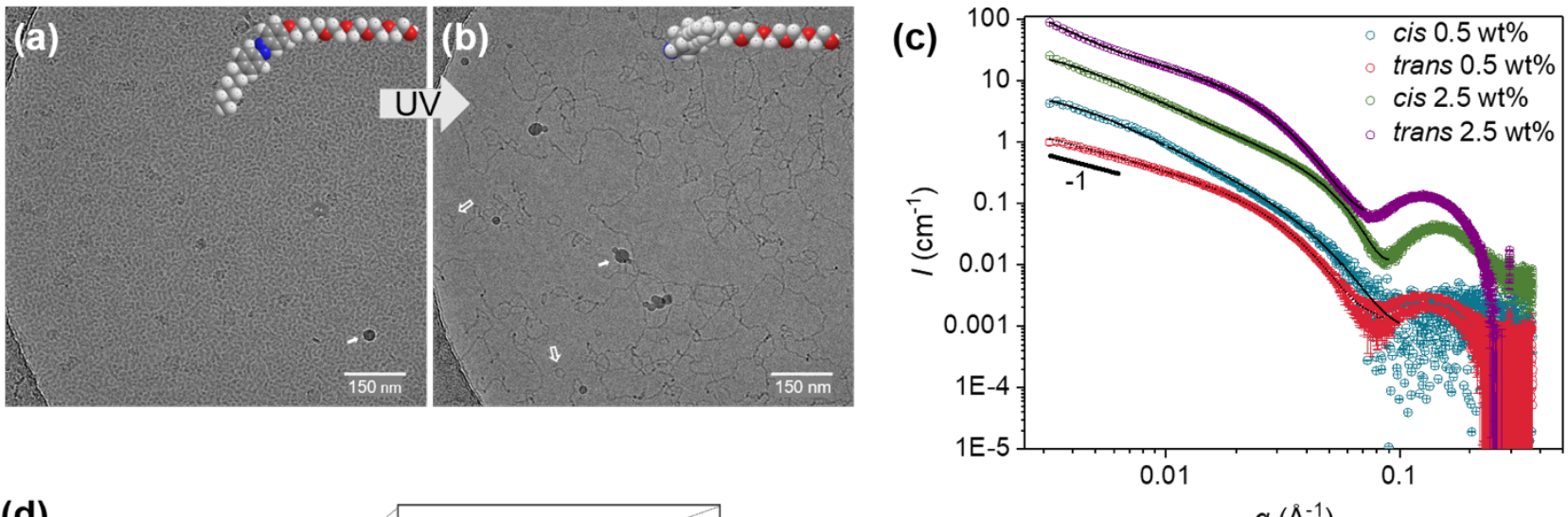

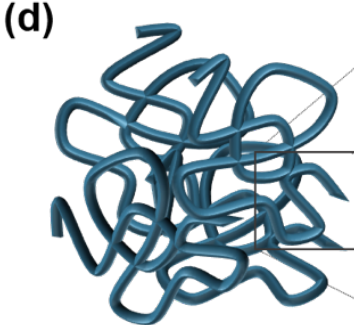

\section{Entangled Network}

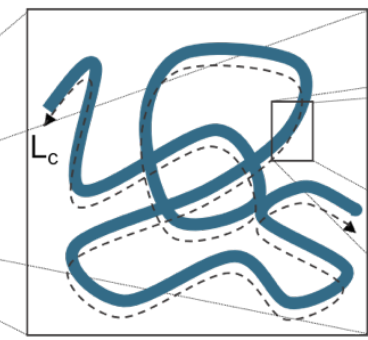

Wormlike Micelle

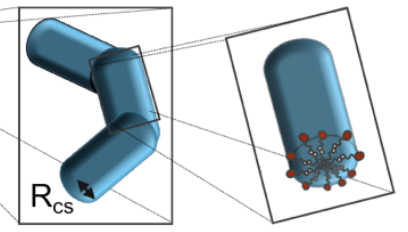

Constituent

\section{Monomer Assembly}

$q\left(\AA^{-1}\right)$

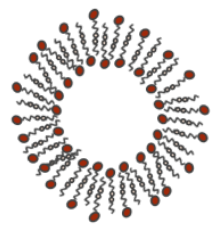

Vesicle

Figure 3. Structural analysis of $\mathrm{C}_{6} \mathrm{AzoOC}_{4} \mathrm{E}_{4}(0.5 \mathrm{wt} \%$ in water unless indicated). Cryo-TEM images in (a) the native trans state and (b) after 90 minutes UV irradiation. The scale bar represents $150 \mathrm{~nm}$. The solid white arrows indicate artefacts of the vitrification process, while open arrows indicate vesicles. Insets show 3D structure of the AzoPS monomer in the trans and cis isomeric forms, generated using the 3-D modelling software Chem3D. (c) SAXS profile of $\mathrm{C}_{6} \mathrm{AzoOC} \mathrm{C}_{4} \mathrm{E}_{4}$ before $(0.5$ wt $\%$, red circles, 2.5 wt\%, purple circles) and after ( $0.5 \mathrm{wt} \%$, blue circles, $2.5 \mathrm{wt} \%$, green circles) UV illumination. The short black line indicates a decay of -1 . Fits to the data are indicated by a dotted line (cylinder model) and a solid line (flexible cylinder model). (d) Pictorial representation of an entangled micelle network, a single wormlike micelle and its constituent cylinders comprised of surfactant monomers and a vesicle.

radius: $42.0 \AA ̊$; Kuhn length: $177.2 \AA$ ). No single model or structure factor contribution was found that considered the interference maxima at $0.1 \AA$, although it is possible a combination of models may account for it (ESI, Figure S3). Therefore, for clarity, model fittings have only been shown to $0.09 \AA$ A. Comparisons of the fits obtained using different cylinder models to the scattering profiles can be found in the ESI (Figure S3). All structural parameters obtained from model fitting can be found in the ESI (Table S3). These conclusions can also be reached from examination of the cryo-TEM images, where on the tens of $\mathrm{nm}$ scale, the self-assembled structures appear similar (threadlike). The most significant differences between the micellar networks seem to emerge outside the observation window of a SAXS measurement, on the scale of $n m-\mu m$. As the nanostructure in Figure $3 \mathrm{~b}$ corresponds to a solution with a viscosity close to that of pure water with no shear-thinning behaviour, taking into consideration the cryo-TEM images, packing parameter predictions and SAXS profiles, the change in flow behaviour must be attributed to a change in the degree of entanglement between the wormlike micelles, rather than a dramatic change in the self-assembled structure (e.g. a complete WLM to spherical transition). The linear, planar transisomer can pack effectively, with possible $\pi$ - $\pi$ stacking between azobenzene units and $\mathrm{H}$-bonding between the ethylene glycol units of neighbouring head groups. These interactions would favour longer chain lengths and less branching for this more hydrophobic isomer. In fact, the ability of the trans-AzoPS to pack effectively has been shown to result in highly-organised structures at high concentrations, such as lyotropic liquid crystal phases. ${ }^{44,45}$ Upon photoisomerisation to the bent, non-planar, more hydrophilic cis-isomer, this packing ability is inhibited. A disruption in packing and self-assembly behaviour upon photoisomerisation has been previously reported for azobenzene derivatives, generally attributed to a change in molecular geometry and polarity. ${ }^{43-45,68}$ In this case, the offset in packing ability is expected to lead to an increased number of kinks, twists and branching in the cis-dominant solution, as observed in Figure $3 \mathrm{~b}$. The resulting lower-density network allows for greater force dissipation than the extremely entangled network observed for the trans-dominant solution. For the cis-dominant solution, the presence of vesicles, joints and branching within the micellar network would allow sliding along the WLM length, preventing the formation of the highly entangled network that results in a correspondingly high viscosity and viscoelastic behaviour. Often, the mechanism attributed to responsive, rheological behaviour is a complete change in packing behaviour upon application of a stimulus e.g. a full conversion from wormlike to spherical transition ${ }^{1,2,46}$ or a substantial shortening of WLMs and subsequent reduction in relaxation time. ${ }^{8,34}$ The results here emphasise that the behaviour of the micellar network on a larger scale (e.g. 
presence of branching, degree of entanglement) is also a key parameter for controlling the rheological behaviour.

\section{Conclusions}

In conclusion, we have demonstrated that a neutral AzoPS, $\mathrm{C}_{6} \mathrm{AzoOC}_{4} \mathrm{E}_{4}$, can be used to create a simple, salt-free, lightresponsive fluid, at reasonably low concentrations in water $(2.5$ wt\%), with a viscosity and viscoelasticity that can be reversibly controlled using light. Here, a viscosity difference of four orders of magnitude is achieved before and after UV irradiation, with the initial high-viscosity state recoverable with blue light illumination. Cryo-TEM images and SAXS data suggest the formation of a densely entangled network of wormlike micelles for the native trans-isomer solution, in agreement with the observed birefringence and shear-thinning behaviour. Interestingly, upon UV irradiation the long cylindrical micelles persist (with some vesicle formation), albeit with a different, looser network structure (branching, kinks, twists present). This result can be used to inform the design of further rheological fluids, based on merely disrupting the network structure, rather than drastically and completely changing the self-assembled structure formed by the constituent surfactant molecules. $\mathrm{C}_{6} \mathrm{AzoOC}_{4} \mathrm{E}_{4}$ shows promise in applications that require a dramatic switch in flow behaviour e.g. alternating between drag-reduction and efficient heat-transfer capabilities for heating and cooling applications. ${ }^{13}$ In particular, applications based on stimuli-responsive materials require that the switch between properties be significant, such as the four order of magnitude difference in viscosity achieved here. Exciting, future research opportunities lie in integrating simple, photorheological fluids, like $\mathrm{C}_{6} \mathrm{AzoOC}_{4} \mathrm{E}_{4}$, with microfluidic device technology, and sensing and controlled-release applications.

\section{Conflicts of interest}

There are no conflicts to declare.

\section{Acknowledgements}

We acknowledge Diamond Light Source for time on Beamline B21 under Proposal SM16235-2. EAK thanks the Winton Programme for the Physics of Sustainability and the EPSRC (EP/N509620/1) for financial support. This work was supported in part by an Isaac Newton Trust/University of Cambridge Early Career Support Scheme grant. RD and NWF acknowledge the EPSRC (EP/S009000/1) for financial support. GD acknowledges funding from the Horizon 2020 grant agreement No. 823717 ESTEEM3 and thanks Dr Kasim Sader and the Cambridge Pharmaceutical Cryo-EM Consortium (Thermo Fisher Scientific) for use of the Krios TEM. This work benefited from the use of the SasView application, originally developed under NSF award DMR-0520547. SasView also contains code developed with funding from the European Union's Horizon 2020 research and innovation programme under the SINE2020 project, grant agreement No. 654000.

\section{References}

1 H. Oh, A. M. Ketner, R. Heymann, E. Kesselman, D. Danino, D. E. Falvey and S. R. Raghavan, Soft Matter, 2013, 9, 5025-5033.

A. M. Ketner, R. Kumar, T. S. Davies, P. W. Elder and S. R. Raghavan, J. Am. Chem. Soc., 2007, 129, 1553-1559.

K. Tsuchiya, Y. Orihara, Y. Kondo, N. Yoshino, T. Ohkubo, H. Sakai and M. Abet, J. Am. Chem. Soc., 2004, 126, 1228212283.

Z. Chu and Y. Feng, Chem. Commun., 2011, 47, 7191-7193.

R. Yang, S. Peng and T. C. Hughes, Soft Matter, 2014, 10, 2188-96. Wu, S. Fang and W. Lv, Langmuir, 2017, 33, 4319-4327. T. S. Davies, A. M. Ketner and S. R. Raghavan, J. Am. Chem. Soc., 2006, 128, 6669-6675.

8 R. Kumar, A. M. Ketner and S. R. Raghavan, Langmuir, 2010, 26, 5405-5411.

9 J. Myska and Z. Chara, Exp. Fluids, 2001, 30, 229-236. K. Gasljevic and E. F. Matthys, Energy Build., 1993, 20, 4556.

11 D. Ohlendorf, W. Interthal and H. Hoffman, Rheol. Acta, 1986, 486, 468-486.

I. Harwigsson and M. Hellsten, J. Am. Oil Chem. Soc., 1996, 73, 921-928.

13 H. Shi, W. Ge, H. Oh, S. M. Pattison, J. T. Huggins, Y. Talmon, D. J. Hart, S. R. Raghavan and J. L. Zakin, Langmuir, 2013, 29, 102-109.

14 H. Shi, Y. Wang, B. Fang, X. Y. Talmon, W. Ge, S. R. Raghavan and J. L. Zakin, Langmuir, 2011, 27, 5806-5813. H. Afifi, G. Karlsson, R. K. Heenan and C. A. Dreiss, J. Colloid Interface Sci., 2012, 378, 125-134.

16 J. Zhang, L. Wang, H. Wang and K. Tu, Biomacromolecules, 2006, 7, 2492-2500.

C. Rodriguez-abreu, K. Aramaki, Y. Tanaka and M. A. Lopezquintela, J. Colloid Interface Sci., 2005, 291, 560-569.

18 M. Crothers, Z. Zhou, M. P. S. Ricardo, Z. Yang, P. Taboada, C. Chaibundit, D. Attwood and C. Booth, Int. J. Pharm., 2005, 293, 91-100. P. Dalhaimer, A. J. Engler, R. Parthasarathy and D. E. Discher, Biomacromolecules, 2004, 1714-1719. S. Avramiotis, V. Papadimitriou, E. Hatzara, V. Bekiari, P. Lianos and A. Xenakis, Langmuir, 2007, 23, 4438-4447.

21 K. Bohon and S. Krause, J. Polym. Sci. Part B Polym. Phys., 1997, 36, 1091-1094.

J. D. Kretlow, L. Klouda and A. G. Mikos, Adv. Drug Deliv. Rev., 2007, 59, 263-273. T. Wang, J. Jiang, Y. Liu, Z. Li and M. Liu, Langmuir, 2010, 26, 18694-18700.

M. Cho, J. Kim, H. J. Choi and S. Choi, Smart Mater. Struct., 2017, 26, 054007. W. J. Kim and S. M. Yang, Langmuir, 2000, 16, 4761-4765. B. Tan, A. Dozier, H. Lehmler, B. L. Knutson and S. E. Rankin, Langmuir, 2004, 6981-6984.

27 G. Li and Z. Zhang, Macromolecules, 2004, 37, 2683-2685.

28 S. Nagamine and K. Kurumada, Adv. Powder Technol., 
2001, 12, 145-156.

J. Yang, Z. Yang, Y. Lu, J. Chen and W. Qin, J. Dispers. Sci. Technol., 2013, 34, 1124-1129. V. Croce, T. Cosgrove, C. A. Dreiss, G. Maitland and T. Hughes, Langmuir, 2004, 20, 9978-9982.

Y. Z. Dong, Y. Seo and H. J. Choi, Soft Matter, 2019, 15, 3473-3486. Y. P. Seo, S. Han, J. Choi, A. Takahara, H. J. Choi and Y. Seo, Adv. Mater., 2018, 30, 1-13.

H. Sakai, Y. Orihara, H. Kodashima, A. Matsumura, T. Ohkubo, K. Tsuchiya and M. Abe, J. Am. Chem. Soc., 2005, 127, 13454-13455. H. Y. Lee, K. K. Diehn, K. Sun, T. Chen and S. R. Raghavan, J. Am. Chem. Soc., 2011, 133, 8461-8463. H. Sakai, H. Ebana, K. Sakai, K. Tsuchiya and T. Ohkubo, J. Colloid Interface Sci., 2007, 316, 1027-1030.

C. T. Lee, K. A. Smith and T. A. Hatton, Macromolecules, 2004, 37, 5397-5405.

G. Pouliquen and C. Tribet, Macromolecules, 2006, 39, 373-383.

Y. Yang, H. Kim, A. Fridman and Y. I. Cho, Int. J. Heat Mass Transf., 2010, 53, 412-422.

S. N. Kazi, G. G. Duffy and X. D. Chen, Desalination, 2012 288, 126-134.

B. O. Hasan, G. J. Nathan, P. J. Ashman, R. A. Craig and R. M. Kelso, Appl. Therm. Eng., 2012, 36, 210-218. $\mathrm{H}$. Schnablegger and Y. Singh, The SAXS Guide, Anton Paar GmbH, Graz, Austria, 3rd edn., 2013. C. Blayo, J. E. Houston, S. M. King and R. C. Evans, Langmuir, 2018, 34, 10123-10134.

E. A. Kelly, J. E. Houston and R. C. Evans, Soft Matter, 2019, 15, 1253-1259.

J. E. Houston, E. A. Kelly, M. Kruteva, K. Chrissopoulou, N. Cowieson and R. C. Evans, J. Mater. Chem. C, 2019, 7, 10945-10952.

S. Peng, Q. Guo, T. C. Hughes and P. G. Hartley, Langmuir, 2014, 30, 866-872.

B. Song, Y. Hu and J. Zhao, J. Colloid Interface Sci., 2009, 333, 820-822.

http://www.sasview.org/, accessed 10/19.

C. A. Dreiss, Soft Matter, 2007, 3, 956-970.

Z. Chu, C. A. Dreiss and Y. Feng, Chem. Soc. Rev., 2013, 42, 7174.

P. Schurtenberger, R. Scartazzini and P. L. Luisi, Rheol. Acta, 1989, 28, 372-381.

Y. Lin, X. Cheng, Y. Qiao, C. Yu, Y. Yan and J. Huang, Soft Matter, 2010, 6, 902-908.

S. R. Raghavan and E. W. Kaler, Langmuir, 2001, 17, 300306.

R. Kumar, G. C. Kalur, L. Ziserman, D. Danino and S. R. Raghavan, Langmuir, 2007, 12849-12856.

J. Wang, Y. Feng, N. R. Agrawal and S. R. Raghavan, Phys. Chem. Chem. Phys., 2017, 19, 24458-24466.

D. P. Acharya and H. Kunieda, Adv. Colloid Interface Sci., 2006, 123-126, 401-413.

M. E. Cates, Macromolecules, 1987, 20, 2289-2296.

J. N. Israelachvili, D. J. Mitchell and B. W. Ninham, J. Chem.
Soc. Faraday Trans. 2 Mol. Chem. Phys., 1976, 72, 15251568.

58 Y. Takahashi, M. Kishimoto and Y. Kondo, J. Colloid Interface Sci., 2016, 470, 250-256.

59 T. P. T. Dao, A. Brûlet, F. Fernandes, M. Er-Rafik, K. Ferji, R. Schweins, J. P. Chapel, A. Fedorov, M. Schmutz, M. Prieto, O. Sandre and J. F. Le Meins, Langmuir, 2017, 33, 17051715.

60 J. S. Pedersen and P. Schurtenberger, Macromolecules, 1996, 29, 7602-7612.

61 M. J. Derry, O. O. Mykhaylyk and S. P. Armes, Angew. Chemie - Int. Ed., 2017, 56, 1746-1750. M. J. Derry, L. A. Fielding, N. J. Warren, C. J. Mable, A. J. Smith, O. O. Mykhaylyk and S. P. Armes, Chem. Sci., 2016, 7, 5078-5090.

63 C. J. Mable, L. A. Fielding, M. J. Derry, O. O. Mykhaylyk, P. Chambon and S. P. Armes, Chem. Sci., 2018, 9, 1454-1463. R. Garcia-Diez, C. Gollwitzer and M. Krumrey, J. Appl. Crystallogr., 2015, 20-28.

S. Disch, The Spin Structure of Magnetic Nanoparticles and in Magnetic Nanostructures, p. 91, 105, 117, 154 Forschungzentrum Juelich GmBH, 2010. L. A. Feigin and D. I. Svergun, Structure Analysis by Small Angle X-ray and Neutron Scattering, p. 99, Plenum, New York, 1987. R. Pecora and R. Borsali, Soft Matter Characterization, p. 759springer, 2008.

S. Peng, Q. Guo, P. G. Hartley and T. C. Hughes, J. Mater Chem. C, 2014, 2, 8303-8312. 